\title{
NUMERICAL STUDY OF THE RENORMALIZATION GROUP EQUATIONS IN THE FOUR-CELL APPROXIMATION
}

\author{
J.A. TJON \\ Institute for Theoretical Physics, University of Utrecht, The Netherlands
}

Received 16 August 1974

\begin{abstract}
The critical surface is determined for the two-dimensional Ising system of the triangular lattice using the four-cell approximation. In particular, it is shown that for sufficiently large values of the four-spin interaction it is possible to have an anti-ferromagnetic transition.
\end{abstract}

Besides the $\epsilon$-expansion approach [1] to critical phenomena, very encouraging results have been obtained recently by Niemeyer and Van Leeuwen [2] in support of the renormalization group ideas of Wilson and Kadanoff. Using finite clusters of cells in the two-dimensional Ising system of a triangular lattice they constructed renormalization group (r.g.) equations for the Ising system and found that they give rise to a fixed point. The resulting critical exponents are in good agreement with the known analytic results. The purpose of this note is to study in detail the solutions to these equations in the four-cell approximation. In particular results are presented on the nature of the surface of criticality which in most previous studies have been assumed for simplicity to be almost flat.

In the four-cell approximation the reduction from the original spin system to the cell system give in the absence of a magnetic field r.g. equations in three interaction parameters $k_{1}, k_{2}$ and $k_{3}$ representing respectively the nearest neighbour, next to nearest neighbour and the four-spin interaction. The r.g. equations being non-linear are complicated and have to be studied numerically. Only one physical fixed point was found at $k_{1}=0.2933, k_{2}=0.0007, k_{3}=$ $-0.0029^{\ddagger}$. These values are slightly different from ref. [2], because we have adopted here the procedure of averaging the different strengths of the bonds instead of the Ursell expansion. The surface of criticality connected with this fixed point was next determined and found to be consisting of a single surface.

\footnotetext{
\# The relevant eigenvalues found are $\lambda_{T}=1.564$ and $\lambda_{h}=$ 2.317, which are consistent with ref. [2].
}

Since in the absence of the four-spin interaction only a ferromagnetic phase transition is possible it is expected that for the critical line $k_{2}$ should decrease with increasing $k_{1}$. This was indeed found to be the case. For $k_{1}>0$ and $1>\alpha=k_{2} / k_{1}>0$ the line is almost straight while for negative $\alpha$ there is considerably more curvature. The value of the slope for $\alpha<1$ is given by $m=1.20$ which is consistent with ref. [2] and should be compared with $m=1.35$ of Dalton and Wood [3].

In the presence of $k_{3}$ the situation can be quite different. In fig. 1 a perspective plot is shown of the



Fig. 1. Perspective plot of the surface of criticality $k_{2}\left(k_{1}, k_{3}\right)$. 




(a)

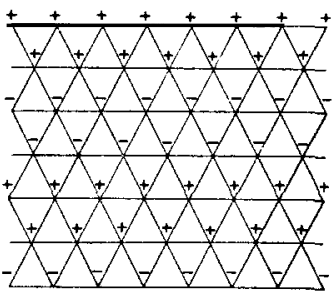

(b)
Fig. 2. (a) Antiferromagnetic state which is dominant for $k_{1} \ll 0$ and $k \gg 0$. (b) Antiferromagnetic state which is dominant for $k_{1} \gg 0$ and $k_{3} \ll 0$.

critical surface of $k_{2}$ as a function of $k_{1}$ and $k_{3}$. The values of $k_{1}$ and $k_{3}$ range between -2 and 2 , while $k_{2}$ can be between -1.75 and 2.95 . In the lower right part of fig. 1 we see the typical ferromagnetic behaviour, while for $k_{1}<0$ and $k_{3}>0$ and large, the value of $k_{2}$ increases as function of $k_{3}$ which is indicative for an anti-ferromagnetic state. For large values of $k_{3}$ it is indeed in principle possible to have two types of anti-ferromagnetic states. These are shown in fig. 2. For $k_{1}<0$ and $k_{3}>0$ and large it is expected that state (2a) is dominant, while for $k_{3}<0, k_{1}>0$ and large state (2b) should be dominating. From fig. 1 we see some eivdence for the latter state in the lower left part of the figure. The different regions are separated by ridges in the landscape of the surface of criticality.

Finally, with the aid of the inhomogeneous scaling equations for the free energy, the thermodynamic quantities can be obtained readily using the r.g. equations [4]. The results for the internal energy $\mathrm{d} f / \mathrm{d} k_{1}$ and heat capacity $k_{1}^{2} \mathrm{~d}^{2} f / \mathrm{d} k_{1}^{2}$ for the Ising system with $k_{2}=k_{3}=0$ are shown in fig. 3 . For comparison the exact results [5] and the finite lattice results for twelve spins are also shown. We see that the r.g. equations give a better representation for the thermodynamic quantities than the finite lattice results. On the other hand, the agreement with the exact solutions is worse than that obtained in ref. [4] for the case of the square lattice, studied also in the fourcell approximation. It is clear that in order to find the thermodynamic quantities detailed and accurate knowledge is needed of the r.g. equations and hence it should be concluded that in the four-cell approximation the r.g. equations are not as good for the tri-

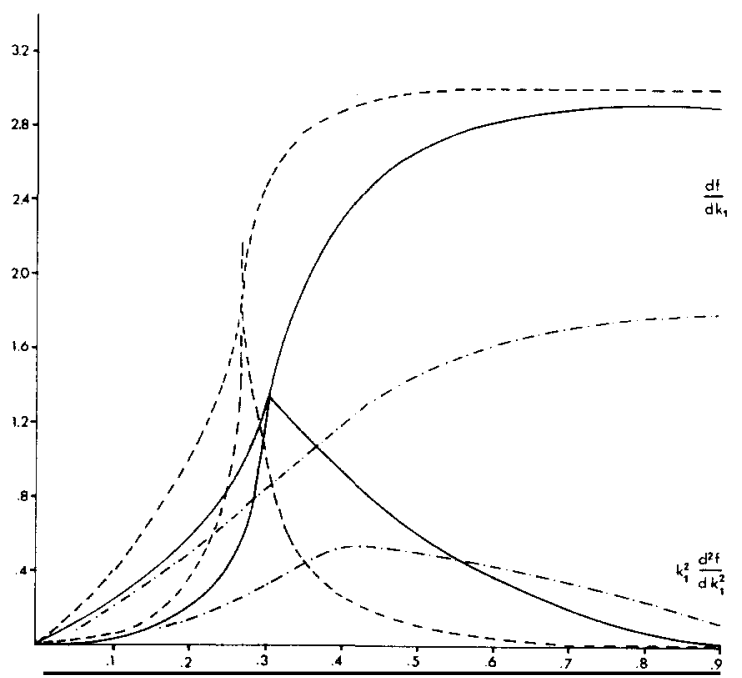

Fig. 3. The internal energy and specific heat of the Ising system. lutions; - . - finite lattice solutions for twelve spins arranged in the four-cell configuration.

angular lattice as for the square lattice. Going to the seven-cell configuration where the critical exponents are in better agreement with the exact results and where periodic boundary conditions can be taken into account, the results for the thermodynamic quantities are expected to improve, but the nature of the surface if criticality as discussed here would essentially not change.

We would like to thank Dr. M. Nauenberg for his help during the initial stages of this work.

\section{References}

[1] For a general review see K. Wilson and J. Kogut, Phys. Rep., to be published.

[2] Th. Niemeyer and J.M.J. Van Leeuwen, Phys. Rev. Lett. 31 (1973) 1412; Physica 71 (1974) 17.

[3] N.W. Dalton and D.W. Wood, J. Math. Phys. 7 (1969) 1271 .

[4] M. Nauenberg and B. Nienhuis, Renormalization group approach to the solution of general Ising systems, preprint.

[5] R.M.F. Houtappel, Physica 16 (1950) 425. 